\title{
Characteristic scales of earthquake rupture from numerical models
}

\author{
M. H. Heimpel \\ University of Alberta, Department of Physics, Edmonton, AB T6G 2J1, Canada
}

\begin{abstract}
Numerical models of earthquake rupture are used to investigate characteristic length scales and size distributions of repeated earthquakes on vertical, planar fault segments. The models are based on exact solutions of static three-dimensional (3-D) elasticity. Dynamical rupture is approximated by allowing the static stress field to expand from slip motions at a single velocity. To show how the vertical fault width affects earthquake size distributions for a broad range of fault behaviors, two different fault strength models are used; a smooth model and a heterogeneous asperity model. The smooth model is a simplified version of the Dieterich-Ruina rate and state dependent friction law. The heterogeneous asperity model uses a slip-dependent random powerlaw strength distribution. It is shown that the characteristic scale of fault segmentation is proportional to the vertical width of a seismogenic fault. This conclusion holds for both the smooth and the heterogeneous models. For the smooth models characteristic quake distributions result, with populations of large events that are obviously distinct from smaller events. The distributions of large events have well-defined mean lengths and moments. The heterogeneous models result in Gutenberg-Richter (GR) powerlaw distributions of event sizes up to a characteristic quake size. Quakes larger than the characteristic size fall off the GR distribution such that the powerlaw would greatly overestimate the probability of occurrence of the larger events.
\end{abstract}

\section{Introduction}

In this paper we investigate how the width and length affects earthquake scaling on shallow faults. It is well known that the powerlaw moment-frequency scaling implied by the GR relation indicates that the earthquake rupture process is scale invariant (Andrews, 1980; Rundle, 1989). Thus, it follows that deviations from the GR relation, which are expected, by

Correspondence to: M. H. Heimpel

(mheimpel@phys.ualberta.ca) physical and geometrical constraints, at both small and large ends of earthquake size-frequency distributions, indicate the effect of characteristic scales of the fault or fault system.

There is a large body of previous literature on earthquake scaling, with contributions from observational, experimental, theoretical and numerical work. Several studies have shown that, for large shallow earthquakes, where the lateral extent of seismogenic slip becomes larger than the seismogenic thickness, the size distribution changes, such that the probability of a larger event is lower than that inferred from smaller quakes (e.g. Pacheco et al., 1992; Okal and Romanowics, 1994; Scholz, 1994). Lateral fault terminations also put constraints on the shape of earthquake size distributions. A simple example is an oceanic transform fault, bounded by midocean ridge segments. In that case, a scaling change is associated with the maximum magnitude, which is characteristic of the fault area (Hyndman and Weichert, 1983). Smaller scale fault discontinuities, as well as jogs and bends have been shown put strong constraints on rupture propagation and arrest (Nielsen and Knopoff, 1998).

Paleoseismology provides useful information on large earthquakes over several earthquake cycles. Models based on paleoseismicity data emphasize the recurrence of large events that are characteristic of the fault system geometry (e.g. Schwartz and Coppersmith, 1984; Wesnousky, 1994; Sieh, 1996). In those studies characteristic events are indicated by a local peak in the earthquake size-frequency distribution. The well-studied earthquake data on the Parkfield segment of the San Andreas fault, which features powerlaw moment-frequency scaling for small events and a quasiperiodic sequence of moderately large events, represent a good example of charactecteristic earthquake behaviour over a relatively short time scale (e.g. Ben Zion and Rice, 1993).

Various types of numerical models that result in synthetic earthquake catalogues have previously been used to investigate earthquake scaling. These include mass-spring slider models (Burridge and Knopoff, 1967; Carlson and Langer, 1989), cellular automaton and rule-based models (Gross, 1996; Steacy and McClosky, 1999), quasistatic elas- 
Table 1. List of symbols

\begin{tabular}{|c|c|c|}
\hline Notation & & Units \\
\hline$A$ & fault area & $\mathrm{m}^{2}$ \\
\hline$G$ & shear modulus & $\mathrm{N} \mathrm{m}^{-2}$ \\
\hline$K$ & rate-state friction constant & $*$ \\
\hline$L$ & rupture length & $\mathrm{m}$ \\
\hline$L^{*}$ & dimensionless rupture length & $*$ \\
\hline$L_{c}$ & rate-state friction: critical slip distance & $\mathrm{m}$ \\
\hline$L_{h}$ & heterogeneous friction: mean slip distance & $\mathrm{m}$ \\
\hline$M$ & seismic moment & $\mathrm{m}^{3}$ \\
\hline$M^{*}$ & dimensionless seismic moment & * \\
\hline$N$ & cumulative number of events & * \\
\hline$P$ & pore pressure & $\mathrm{N} \mathrm{m}^{-2}$ \\
\hline$T$ & tectonic loading time & $\mathrm{s}$ \\
\hline$c$ & stress transfer velocity, shear velocity & $\mathrm{m} \mathrm{s}^{-1}$ \\
\hline$d_{v}$ & nucleation size & $\mathrm{m}$ \\
\hline$g_{i j}$ & stress transfer kernel & $\mathrm{N} \mathrm{m}^{-3}$ \\
\hline$h$ & vertical fault width & $\mathrm{m}$ \\
\hline$k$ & rate-state friction constant & $\mathrm{s}$ \\
\hline$p, q$ & lateral and vertical number of cells & * \\
\hline$s$ & event size & \# \\
\hline$t$ & time & $\mathrm{s}$ \\
\hline$u$ & slip & $\mathrm{m}$ \\
\hline$v_{r}$ & rupture velocity & $\mathrm{m} \mathrm{s}^{-1}$ \\
\hline$v_{e}$ & two-sided horizontal expansion rate & $\mathrm{m} \mathrm{s}^{-1}$ \\
\hline$v_{S}$ & slip velocity & $\mathrm{m} \mathrm{s}^{-1}$ \\
\hline$x$ & lateral coordinate & $\mathrm{m}$ \\
\hline$z$ & vertical coordinate & $\mathrm{m}$ \\
\hline$\alpha$ & modified GR distribution parameter & \# \\
\hline$\beta$ & moment-frequency relation powerlaw exponent & * \\
\hline$\beta_{L^{*}}$ & length-frequency relation powerlaw exponent & $*$ \\
\hline$\chi$ & fault aspect ratio & * \\
\hline$\delta, \delta_{x}, \delta_{y}$ & cell size & $\mathrm{m}$ \\
\hline$\gamma$ & modified GR distribution parameter & $*$ \\
\hline$\kappa$ & strength distribution scale parameter & $*$ \\
\hline$\lambda$ & modified GR distribution moment scale parameter & $*$ \\
\hline$\lambda_{L^{*}}$ & modified GR distribution length scale parameter & * \\
\hline$\mu$ & characteristic rupture moment & * \\
\hline$\mu_{L^{*}}$ & characteristic rupture length & $*$ \\
\hline$v$ & mean of frictional strength distribution & * \\
\hline$\phi$ & fault friction coefficient & * \\
\hline$\phi_{0}$ & rate-state friction constant & $*$ \\
\hline$\phi_{f}$ & friction fluctuation & * \\
\hline$\sigma$ & shear stress & $\mathrm{N} \mathrm{m}^{-2}$ \\
\hline$\sigma_{0}$ & tectonic stress & $\mathrm{N} \mathrm{m}^{-2}$ \\
\hline$\sigma_{n}$ & normal stress & $\mathrm{N} \mathrm{m}^{-2}$ \\
\hline$\theta$ & friction state parameter & $\mathrm{s}$ \\
\hline
\end{tabular}

* dimensionless

\# indeterminate units 
tic models (Rice, 1993; Ben-Zion and Rice 1993; Heimpel 1997; Nielsen and Knopoff 1998), and elastodynamic models (Shaw, 2000; Lapusta et al., 2000).

Two types of constitutive models have been employed on numerical faults: heterogeneous or asperity models and smooth, rate and state dependent models. Heterogeneous models attempt to represent the observed geometrical complexity of fault surfaces as heterogeneous properties on a planar fault (Ben-Zion and Rice, 1993; Gross, 1996; Heimpel, 1997; Steacy and McClosky, 1999). All of these result in powerlaw moment-frequency relations, although the powerlaw slope may depend on the details of the fault heterogeneity. Smooth models typically employ a friction formulation based on laboratory experiments of frictional sliding (Dieterich, 1979, 1992; Beeler et al., 1994). Recurring stick slip behaviour may be obtained in the absence of other imposed fault heterogeneity when two necessary conditions are satisfied: (1) friction decreases with increasing slip velocity and (2) healing occurs at very slow and/or zero slip velocity. Large events occur under these conditions if the friction coefficient decreases at a sufficient rate for slip instability. Smooth models with adequate spatial resolution result, for unstable slip events, in only a relatively small range of large event sizes. For smooth models to be adequately resolved, nucleation of unstable slip events must involve a sufficiently large number of numerical cells such that individual cells cannot behave independently. Whereas resolved models are said to have a continuum limit, under-resolved smooth models, may exhibit powerlaw event size distributions due to cell-size effects (Rice, 1993).

\section{Model formulation}

Here, repeating earthquake models are studied, for smooth and heterogeneous friction on planar faults, varying the fault geometry, represented by the aspect ratio $\chi=L / h$, where $L$ is the fault length and $h$ is the vertical fault width. In all the models the faults are vertical and strike slip, and only the shear stresses are considered to vary in the elastic stress transfer.

Elastic stress transfer is calculated with the following generalized equation

$\sigma_{i}(t)=\sum_{j} g_{i j} u_{j}+\frac{\sigma_{0} t}{T}$

where $\sigma_{i}(t)$ is the shear stress at the center of cell $i$ due to slip $u_{j}$ at cell $j$, and $g_{i j}$ is the static elastic stress transfer kernel, which includes free surface and lateral periodic boundary conditions. The second term on the left-hand side of equation 1 is the uniformly increasing tectonic stress, where $\sigma_{0}$ is a reference shear stress, $t$ is time, and $T$ is the tectonic timescale (see Sect. 2.1.1 for a discussion of $T$ ).

The numerical domain is a rectangular plane surface, discretized into $p \times q$ square or rectangular cells. The domain represents a vertical fault plane of width $q \delta_{y}$ and lateral extent $p \delta_{x}$, where $\delta_{y}$ and $\delta_{x}$ are the vertical and lateral cell length, respectively. For 1-D and 2-D fault planes, a fullspace stress transfer kernel is constructed by using 3-D elastic solutions for dislocation-lines (Hirth and Lothe, 1982). The free surface boundary condition is then incorporated into the full-space kernel to obtain $g_{i j}$ (Groves and Bacon, 1970; Maurrisson and Capella, 1974a, b).

The periodic side boundary condition is incorporated into $g_{i j}$ by modifying the stress transfer kernel for an elastic halfspace space with a free surface:

$g_{i j}(x, z)=\sum_{k=-p}^{p} g_{i j}^{\prime}(x+k l, z)$.

where $x$ and $z$ are the horizontal and vertical coordinates, $l$ is the fault length, and $g_{i j}^{\prime}$ is the elastic half-space kernel. The periodic condition is obtained exactly for $p=\infty$. Here, a good approximation is achieved by using $p=10$. A thorough discussion of boundary conditions for this type of model can be found in Rice (1993).

The bottom boundary, which represents the bottom of the seismogenic zone, is open. Since slip occurs only down to the bottom boundary, and stress is transferred in the downward direction across the boundary, the open boundary condition may also be referred to as dissipative. Any stress that is transferred across the bottom boundary is dissipated out of the fault domain into a 3-D elastic half-space.

Two-dimensional arrays of square cells and onedimensional arrays of rectangular cells are used for the 2$\mathrm{D}$ and 1-D models, respectively. The horizontal and vertical sides of the slip cells are screw and edge dislocation lines, respectively. Slip is constant over a single cell and shear stress is calculated at cell centers, thus avoiding stress singularities. Slip is quantized so that, if we normalize the quantum of slip to unity, $g_{i j}$ is the shear stress per unit slip at cell $i$ due to a single quantum of slip at cell $j$. The quantum of slip is scaled to parameters in the friction formulation, which are discussed in Sect. 2.1 below.

The models work like a cellular automaton. The background tectonic stress is increased uniformly over the fault between model quakes. A slip event is initiated when the stress of a numerical cell reaches the yield value, which is defined by the frictional constitutive relation (see Sect. 2.1 below). Each individual slip event causes the corresponding static stress field to expand radially from the slip center at the stress transfer velocity $c$. Individual cells may slip once or many times. Under appropriate frictional conditions, rapid and large scale slip events occur due to cascades of individual sub-events. An event cascade proceeds until the entire fault is at a subcritical stress.

The introduction of $c$ represents an improvement over the formulation of quasistatic stress transfer used in the models of Heimpel (1997). It introduces a fundamental timescale $h / c$, which is the transit time of stress across the vertical width $h$ of the fault, eliminates unphysical spatial ordering of sequences of rupture sub-events, and results in a more accurate representation of the rupture propagation process. The rupture propagation velocity $v_{r}$, which arises naturally as the 
result of an event cascade, may have a range of values, such that $v_{r} \leq c$. Since only shear stress is variable in these models, $c$ is analogous to a shear wave velocity. It is noted that, although the transfer of stress and associated friction evolution during slip events in the model is dynamical, the amplitude of stresses neglects inertia. This approximation may be referred as a "pseudo-dynamical approximation" in a similar sense that this phrase has been used by Heaton (1990).

\subsection{Fault constitutive equations}

The geometry of natural faults includes offsets, bending and fault surface topography. Thus, the planar geometry of the fault model represents a drastic approximation with respect to natural faults and fault systems. Two major consequences of this approximation are (1) variations in normal stress are neglected and (2) fault heterogeneity must be introduced via an imposed friction model. Both of these consequences are important in considering the meaning of the formulation of the fault constitutive equation. In fact, the constitutive models chosen here are acknowledged not to be realistic representations of fault rheology. Rather, they are chosen to give relatively simple end-member behaviour, so that the scale of earthquakes and fault segmentation may be identified as a function of the fault dimensions. We study two different friction models. Both the smooth model and the heterogeneous model result in repeating earthquakes and an asymptotic steady state (i.e. constant time- and space-averaged stress), which is obtained after several quake cycles. The smooth model yields a quasi-periodic sequence of large events that have a well defined mean size. This behavior is similar to that of the characteristic earthquake model. The heterogeneous model yields powerlaw size distributions with an exponent that is consistent with the Gutenberg-Richter distribution.

\subsubsection{Smooth friction}

For the smooth model rate and state dependent friction is used. Friction is defined as the ratio of shear and effective normal stress $\phi=\sigma /\left(\sigma_{n}-P\right)$, where $\sigma_{n}$ is the compressive normal stress and $P$ is the pore pressure. Although variations in pore pressure can account for various interesting fault behaviours during an earthquake cycle (e.g. Sleep, 1997; Andrews, 2002), we seek a relatively simple fault constitutive relation, allowing a clear assessment of the effect of fault geometry. Thus, in this paper $\sigma_{n}-P$ is taken to be constant, so that the effect of variations in normal stress are neglected. This condition is valid in the case where pore pressure is proportional to lithostatic pressure and the maximum shear stress direction is parallel to the fault plane. The rate and state dependent fault friction is given by

$\phi=\phi_{0}+K \ln (\theta / k+1)$

$\frac{d \theta}{d t}=1-\theta v / L_{c}$

where $\theta$ is a the state parameter, $k, K$, and $L_{c}$ are constants described below. Equations (3) and (4) are a simplified version of the well-known Dieterich-Ruina friction relation, also

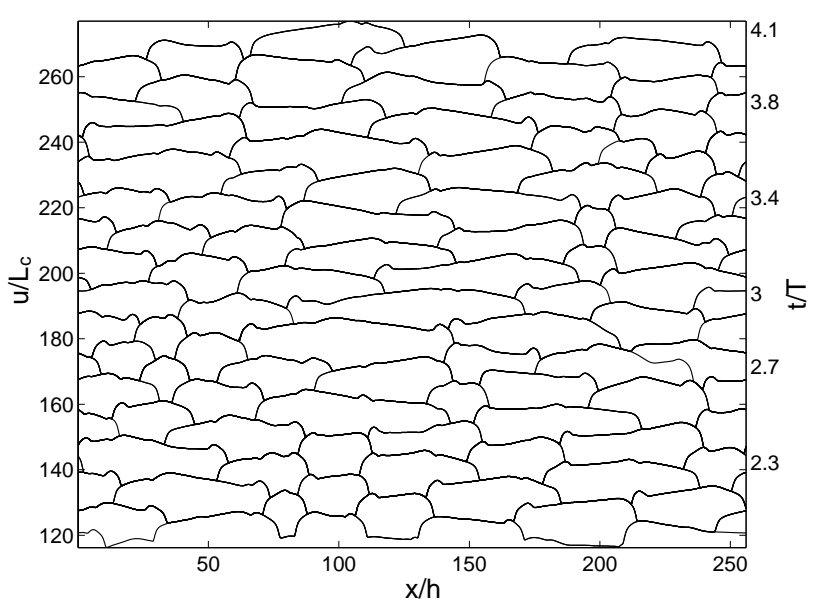

Fig. 1. Depth averaged slip pattern for the smooth fault model. The vertical, two-dimensional fault plane has aspect ratio $\chi=128$. The Numerical domain is composed of $2048 \times 16$ square cells. Fault slip is governed by a simplified version of Dieterich-Ruina rate and state dependent friction (see text for explanation). The pattern is produced by plotting the slip distribution every time a relatively large slip event occurs. Slip $u$, position along the fault $x$, and time $t$ are scaled by $L_{c}, h$ and $T$, respectively.

known as the "slowness" law, where the direct velocity effect has been neglected (Dieterich, 1979, 1992; Beeler et al., 1994). The state parameter $\theta$ has units of time and gives the age of the fault - an older fault has had more time to heal and is thus stronger.

The constant $L_{c}$ has a special significance for the scaling of frictional slip. It is the slip distance over which friction decreases during sudden, rapid slip. In more precise language, it is the slip distance over which the time dependent part of $\theta$ decreases by $1 / e$ of its previous value (as can be seen by integrating Eq. 4). In the models presented here, slip is quantized such that each individual slip has a value of $L_{c} / 500$. In addition, the size of slip cells is set so that the relation $\delta \ll d_{v}$ is satisfied, where $\delta$ is shorter leg of a rectangular cell, and $d_{v}=L_{c} G /\left[\left(\sigma_{n}-P\right) K\right]$, is called the nucleation size, where $G$ is the shear modulus (Rice, 1993; Rice and Ben-Zion, 1996). This assures that, for events of significant magnitude, the model is spatially resolved and the results are independent of the cell size.

In the numerical implementation of Eqs. (1), (3) and (4), two timescales are present; the slip and stress transfer timescale $h / c$, and the tectonic timescale $T$. These timescales correspond to stress changes calculated when the fault is slipping and locked, respectively. During slip events the tectonic stress, which is the second term in Eq. (1), is constant and time $t$ in Eq. (4) represents the stress transfer time. Between events the slip velocity $v=0$ so that Eq. (4) reduces to $d \theta / d t=1$, the first term in Eq. (1) is constant, and $t$ is replaced by the tectonic time $t^{\prime}$. The relationship between the two time variables is $t^{\prime}=t T c / h$, where $T$ scales the inverse rate (or slowness) of background (i.e. tectonic) stress accumulation between rupture events. Here, we 
$\mathrm{u} / \mathrm{L}_{\mathrm{c}}$

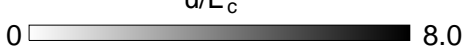

$\mathrm{tc} / \mathrm{h}=9$

$\mathrm{tc} / \mathrm{h}=14$

$\mathrm{tc} / \mathrm{h}=19$

$\mathrm{tc} / \mathrm{h}=24$

tc/h $=29$

$\mathrm{tc} / \mathrm{h}=34$

$\mathrm{tc} / \mathrm{h}=39$

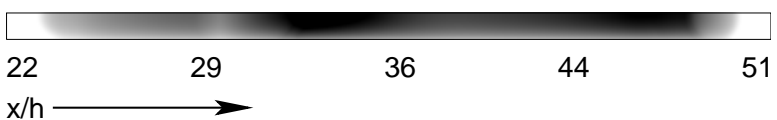

Fig. 2. Development of slip during a rupture event. The cumulative slip at the time of nucleation $(t c / h=0)$ is subtracted out. Elapsed time after nucleation is shown at the top of each successive image. Slip $u$ is scaled by the critical slip distance $L_{c}$. Time $t$ during rupture is scaled by $h / c$, where $c$ is the elastic wave velocity and $h$ is the vertical fault width. The rupture process can be described in three stages: (1) a nucleation stage, with very slow rupture propagation, lasts about 10 time units (top image); (2) break-out, followed by rapid propagation at a large fraction of the elastic wave speed (here, the average horizontal expansion rate $\bar{v}_{e} / c \sim 0.9$ ); (3) arrest, typically occurring at previous arrest sites.

take $T c / h=1 \times 10^{8}$. Using the smooth constitutive relation and taking $c=5 \mathrm{~km} / \mathrm{s}$ as a shear velocity, a model fault scaled to $15 \mathrm{~km}$ width gives $T=3 \times 10^{8} \mathrm{~s}$, and results in a recurrence time of roughly 1 year for large events (see Figs. 1 and 7). This short recurrence interval, which corresponds to a fast background slip rate, represents a numerical compromise balancing realistic fault behaviour and the following time resolution considerations. For very slow tectonic stress accumulation, due to the logarithmic nature of the Dieterich-Ruina friction formulation, the differences in time intervals after an event become very large, making the resolution of time steps impractical. This can be seen by examining Eqs. (3) and (4) during and after a slip event. During a slip event $\theta$ drops as $v>0$ in Eq. (4). When slip ceases, typically $\theta \sim 0$. During the healing phase, $v=0$ in Eq. (4). Combining Eqs. (3) and (4) we obtain $d \phi / d t=K /(\theta+k)$ for the healing phase, were we have used $k=1$. It thus follows that, directly after an event, $\phi \sim \phi_{0}$, and $d \phi / d t$ is maximum. For $T c / h>1 \times 10^{9}$, most of the fault healing can occur over one or a few time steps, thus limiting the
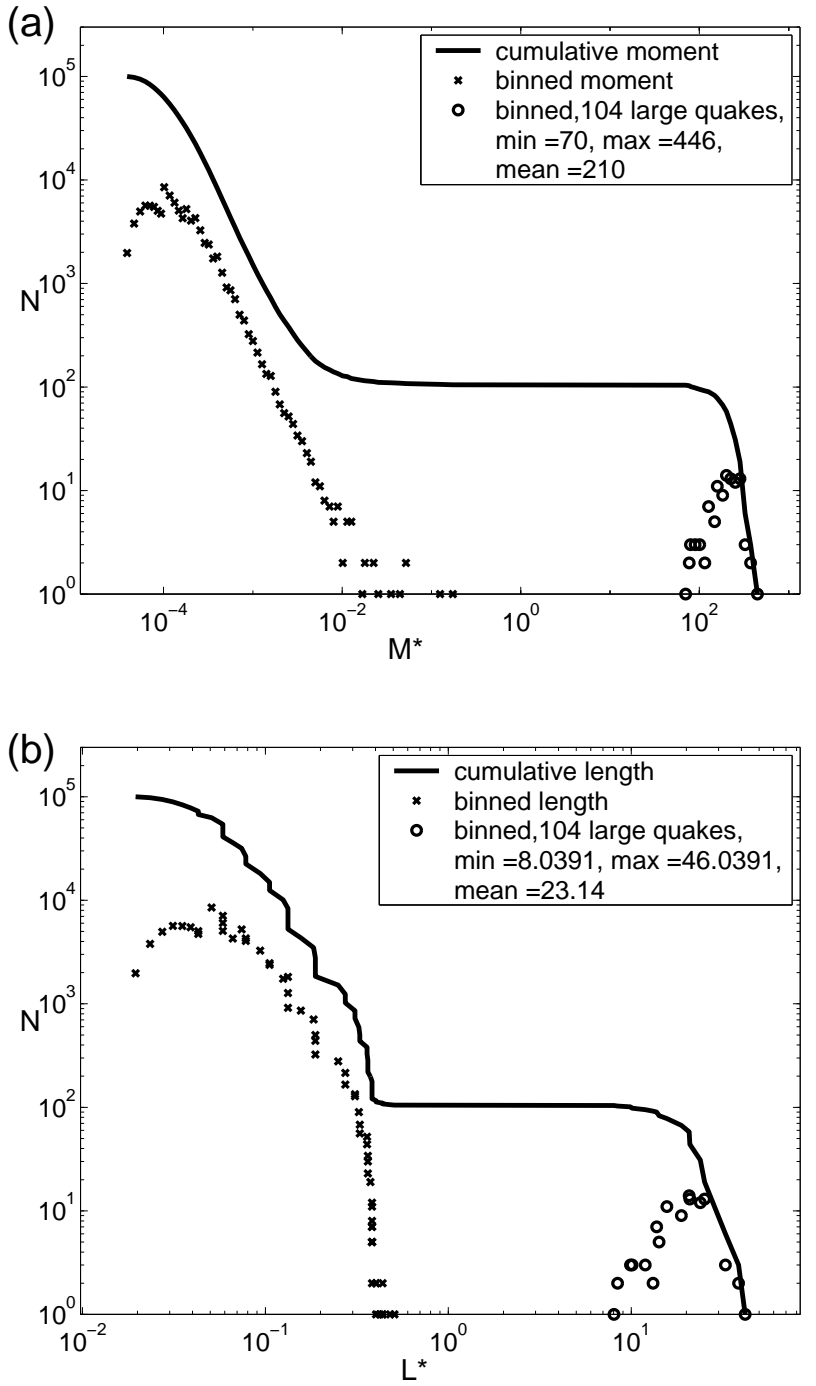

Fig. 3. Event size distributions for the model shown in Figs. 1 and 2. The data interval and bin size for the cumulative and binned distributions respectively is $0.05 \log _{10} M^{*}$, where $M^{*}$ is the dimensionless moment (a), and similarly for the dimensionless rupture length $L^{*}$. Each of the bins for the length distribution (b) contain the same quakes as the corresponding moment bins. Thus the two different quake populations are identical in the moment and length distributions. The smaller events (crosses) are associated with subcritical failure preceding and subsequent to unstable slip events (circles). The unstable slip events involve substantial rupture propagation and account for almost all of the accumulated slip. See the text for an explanation of the moment and length scaling. The distribution of large quakes shown here and in Fig. 1 clearly indicates that the rupture lengths and moments are characteristic of the model system.

resolution of time steps.

For fast tectonic stress accumulation (i.e. small values of $T)$, the earthquake scaling is affected such that the ratio of small to large events increases. Small events are those for which the total slip, and resultant frictional decrease, is insufficient to cause onset of unstable slip. For $T c / h<3 \times 10^{6}$, corresponding to very rapid tectonic loading, the slip is char- 


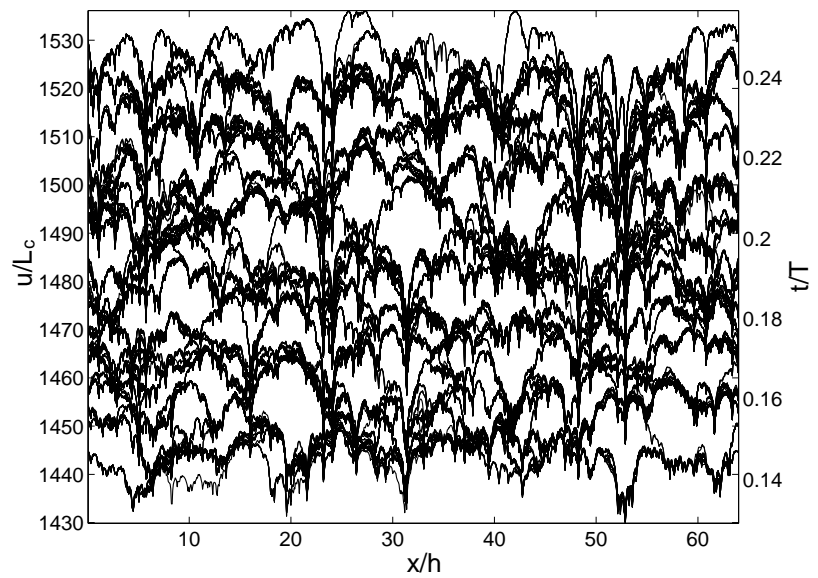

Fig. 4. Depth-averaged slip pattern for the heterogeneous fault model. The model geometry and boundary conditions are similar to the smooth model shown in Fig. 1. Here, fault slip is governed by a simple yield criterion and a highly heterogeneous and random fault strength (see text for details). The aspect ratio is $\chi=64$. The pattern is produced by plotting the slip distribution at equal time intervals $\delta t / T=.0005$.

acterized by a creeping motion, consisting of large numbers of small events and a lack of large events. Although the creeping behavior is interesting, here we focus on earthquake scaling dominated by stick-slip motion. We have found that when sufficiently high values are used, the mean quake size is quite insensitive to variations in $T$ and $10^{7}<T c / h<10^{9}$ gives consistent results.

\subsubsection{Heterogeneous friction}

For the heterogeneous models, the friction is given by

$\phi=\phi_{0}+\phi_{f}$

where $\phi_{f}$ is the fluctuating part of the frictional strength. It is modeled using a yield strength probability distribution (Heimpel, 1996, 1997):

$p\left(\phi_{f}\right)=\frac{2 \phi_{f}}{\kappa^{2}}\left(1+\frac{\phi_{f}^{2}}{\kappa^{2}}\right)^{-2}$

where $\kappa$ is a scale parameter and $v=(\pi / 2) \kappa$ is the mean. Many friction models based on random heterogeneity result in earthquake size distributions with powerlaw statistics. The specific form of this friction model is chosen because it results in synthetic moment-frequency distributions with powerlaw exponent similar to those observed in earthquake moment-frequency distributions. The model is essentially parameterless; $\kappa$ (or $\nu$ ) only changes the mean stress drop, not the shape of size-frequency distributions.

To scale the slip in the heterogeneous models, a critical slip distance $L_{h}$ is defined as the slip distance over which the friction drops, on average, an amount $v$. This is a natural slip distance scale since $v$ is a typical stress drop for large events. Similarly, in the smooth model, ruptures that slip to

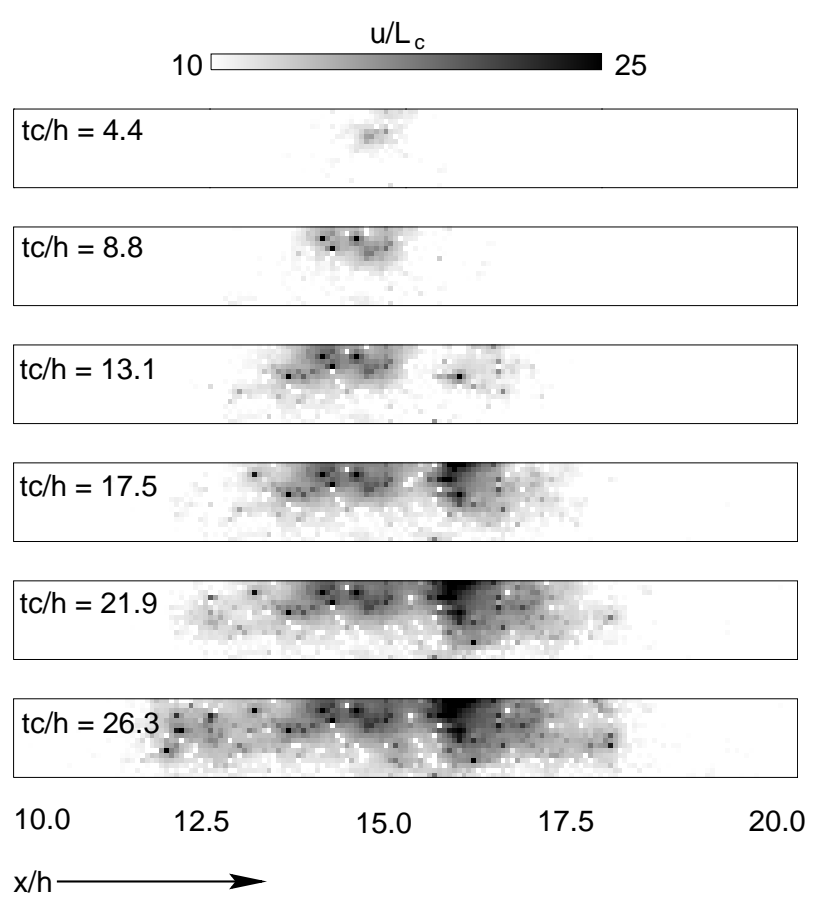

Fig. 5. A "coseismic" slip event for the heterogeneous model. The rupture propagation process is different than that for that smooth model. Here the slip distribution is discontinuous, with weaker parts of the fault yielding early before subsequent slip catches up. The horizontal expansion rate $\bar{v}_{e} / c \sim 0.35$ is more variable and, on average, slower than that for the smooth model.

the critical distance $L_{c}$ correspond to frictional stress drops that result in large events. Also similar to the smooth model, slip is quantized such that each individual slip has a value of $L_{h} / 500$.

The heterogeneous friction model is rule-based. For every quake, each cell that slips at least once is assigned a new strength from Eq. (6) after the initial slip. This new strength value is retained for subsequent slip during the earthquake (Heimpel, 1997). Thus, in contrast to the smooth model, the yield strength formulation allows sudden, appreciable changes in strength, even for a single slip quantum. This means that, in the language of Rice (1993), the heterogeneous model is inherently discrete.

The heterogeneous friction model yields distributions with the following powerlaw exponents: $\beta=2 / 3$ for events expanding in two dimensions on a fault of infinite area (2-D rupture geometry), and $\beta=1 / 2$ for events expanding horizontally on a fault of infinite length and finite width (1-D rupture geometry). Considering standard relations between seismic moment, magnitude and fault area for 1-D and 2-D ruptures, both of the above $\beta$ values correspond to $b=1$ in the GR distribution (Heimpel, 1996). 

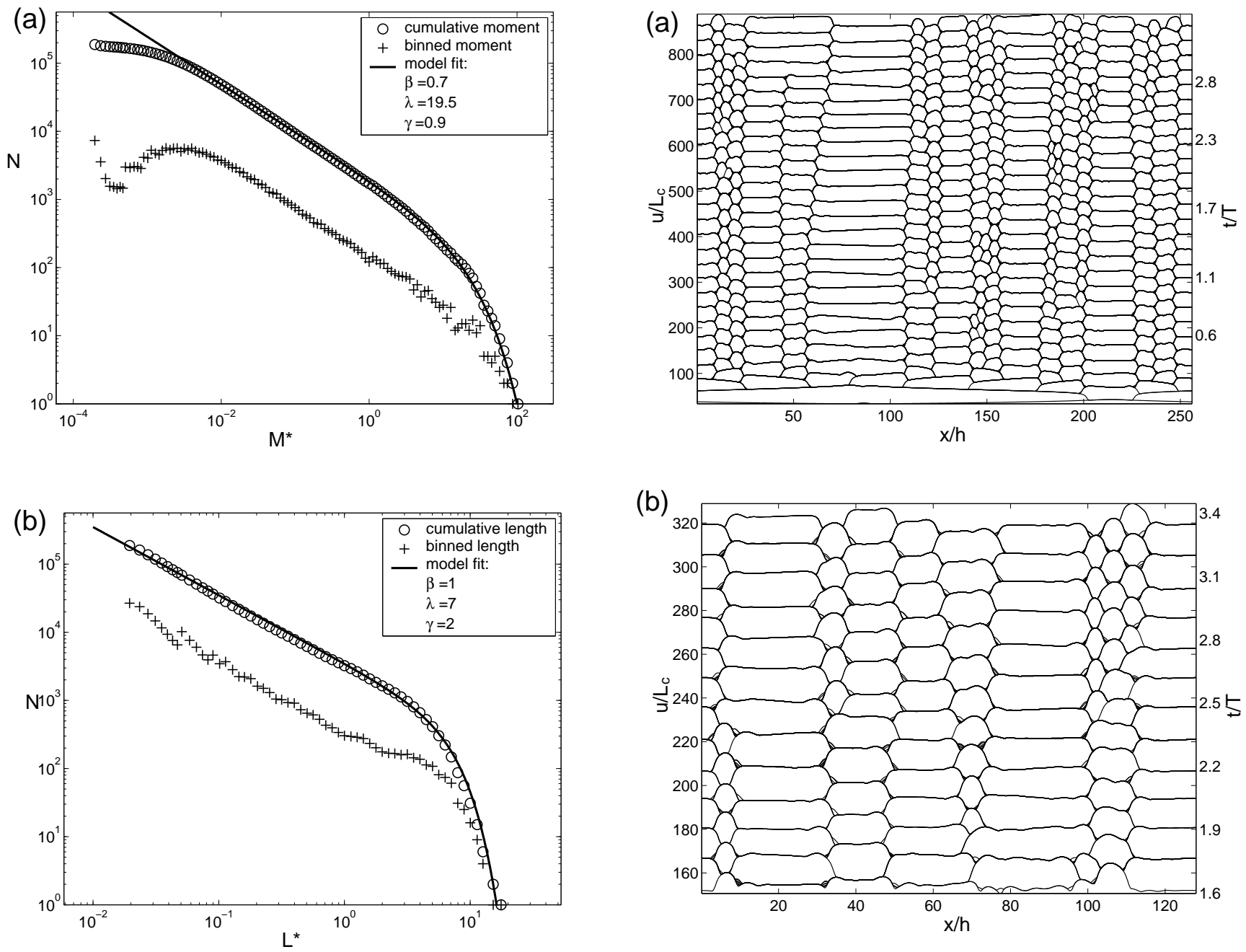

Fig. 6. Event size distributions for the model shown in Figs. 4 and 5. Figures $6 \mathrm{a}$ and $\mathrm{b}$ show results for dimensionless moment $M^{*}$ and dimensionless length $L^{*}$, respectively. For data interval and bin information see Fig. 3. The modified GR relation (see Eq. 7) is used to model the size distributions. A good fit is obtained for values of the parameters $\beta, \lambda$, and $\gamma$, shown in the figures. The size distributions obey a powerlaw for smaller events. Both of the moment and length distributions are consistent with $b \simeq 1$, where $b$ refers to Gutenberg and Richter's b-value. A change in slope occurs for larger events. In Fig. 6b, $\lambda=7$ gives the value where $L^{*}$ falls off the GR distribution. This critical length scale is interpreted here to be characteristic of the fault width.

\section{Results}

\subsection{2-D Fault in an elastic half-space}

For models using the smooth friction model, and aspect ratios smaller than about $\chi=L / h=20$, large model quakes quasi-periodically rupture the entire fault and smaller events do not occur. This happens because, for those models, the characteristic length of large events have an aspect ratio approaching that of the entire fault. In order to obtain repeated ruptures that terminate before rupturing the entire fault we

Fig. 7. Slip pattern for the smooth fault model. The aspect ratios are $\chi=256$ and $\chi=128$ for Figs. 7a and b, respectively. In these models, the vertical fault plane is one-dimensional, and composed of $2048 \times 1$ rectangular cells. As for the model of Fig. 1, fault slip is governed by a simplified version of Dieterich-Ruina rate and state dependent friction. This figure shows that the time of quake recurrence and the length of fault segmentation scales with the fault depth.

must use sufficiently large aspect ratios. The first model fault consists of $2048 \times 16$ square dislocation cells. Thus the aspect ratio of the fault is $\chi=128$. Scaling the model to a shallow fault of $15 \mathrm{~km}$ depth would represent a fault of $1920 \mathrm{~km}$ (i.e. about $50 \%$ longer than the San Andreas fault).

Two calculations are performed for 2-D faults with square slip cells; the first using the smooth friction model and the second using the heterogeneous friction model. Figures 1-3 show results from the smooth model calculation. Figure 1 shows the spatio-temporal slip pattern over about 15 recurrence time intervals for the model fault. This represents about one third of the total calculation time. The slip pattern after several cycles have been completed is indicative of a system that is in an asymptotic steady state, meaning that 
Table 2. Summary of calculations. The unsubscripted parameters $\mu, \beta, \lambda$ and $\gamma$ refer to moment; subscripted parameters refer to length ( $L^{*}$ )

\begin{tabular}{cccccccccccc}
\hline figure(s) & grid size & friction model & $\chi$ & $\mu$ & $\beta$ & $\lambda$ & $\gamma$ & $\mu_{L^{*}}$ & $\beta_{L^{*}}$ & $\lambda_{L^{*}}$ & $\gamma_{L^{*}}$ \\
\hline $1,2,3$ & $2048 \times 16$ & smooth & 128 & 210 & - & - & - & 23.1 & - & - & - \\
$7 \mathrm{a}, 8 \mathrm{a}, 8 \mathrm{c}$ & $2048 \times 1$ & smooth & 256 & 210 & - & - & - & 18.1 & - & - & - \\
$7 \mathrm{~b}, 8 \mathrm{~b}, 8 \mathrm{~d}$ & $2048 \times 1$ & smooth & 128 & 177 & - & - & - & 18.7 & - & - & - \\
\hline $4,5,6$ & $1024 \times 16$ & heterogeneous & 64 & - & 0.7 & 19.5 & 0.9 & - & 1.00 & 7.0 & 2.0 \\
$9 \mathrm{a}$ & $1024 \times 1$ & heterogeneous & 128 & - & 0.52 & 10.0 & 0.50 & - & 1.05 & 7.7 & 1.6 \\
- & $1024 \times 1$ & heterogeneous & 64 & - & 0.50 & 15.0 & 0.50 & - & 0.94 & 7.5 & 1.6 \\
- & $1024 \times 1$ & heterogeneous & 32 & - & 0.49 & 17.0 & 0.60 & - & 0.90 & 8.5 & 1.7 \\
\hline
\end{tabular}

the slip rate and stress, averaged over several quake cycles, is relatively constant.

Nucleation and arrest are controlled by undulations in shear stress, which result from spatially variable slip. We note that the first large event ruptures the entire fault, wraps around and eventually arrests on slight undulations in slip (and stress) formed during nucleation. Subsequent events then tend to arrest on stronger slip undulations that define the terminations of previous events. Slip patterns including smaller events emerge as a result of stress undulations on faults with relatively large aspect ratios. Slip and stress gradients are greatest where ruptures terminate. As a result, rupture terminations are typically identified as the nucleation sites for subsequent ruptures; that result was also observed in numerical models of Shaw (2000). Nucleation and arrest sites may be discerned by looking carefully at Fig. 1. Each event has two rupture terminations, identified as strong slip gradients along the $x / h$ axis. Ruptures are typically asymmetrical (i.e. the rupture front travels primarily either to the left or the right). One or both rupture terminations is frequently close to a previous termination.

The development of slip during a single rupture event is shown in Fig. 2. It is noted that the time-scale in Fig. 2 is different (and much shorter) than that of Fig. 1. The size, duration and character of the event shown is typical of several other large rupture events in this model. Slip in the nucleation area begins at $t=0$. The nucleation phase is well defined and characterized by slip in an area located near a previous rupture termination. Nucleation lasts for about 10 time units (i.e. about $25 \%$ of the total event time). After nucleation the rupture breaks out and accelerates to an average rupture front propagation speed $\bar{v}_{r}$ that is a large fraction of the stress transfer speed $c$. For the event shown in Fig. 2, the faster rupture front travels to the right at $\bar{v}_{r} / c \sim 0.65$. The time-averaged two-sided horizontal expansion rate $\bar{v}_{e} / c \sim 0.9$.

The event moment- and length-frequency distributions are shown in Fig. 3. The dimensionless moment is given by $M^{*}=\frac{M}{h^{2} L_{c}}$, where $M \equiv \bar{u} A$ is defined as the moment, and $\bar{u}$ is the slip, averaged over the rupture area $A$. The rupture length is averaged vertically and scaled horizontally by the vertical fault thickness. Thus, for the 2-D fault models, the average length is $\bar{L}=A / h$ and the dimensionless length is $L^{*}=\bar{L} / h=A / h^{2}$. For the 1-D fault models, the dimensionless length is simply $L^{*}=L / h$ where $L$ is the rupture length. Both moment and length distributions clearly show that, for the smooth friction model, a characteristic event size distribution is obtained. Two distinct populations of event sizes (small and large) are easily identified. The amount of moment accumulated in large events is about 3 orders of magnitude greater than that released in small events. For the population of large events, the depth averaged mean (or characteristic) length is 23.1 fault widths. For a fault of $15 \mathrm{~km}$ width that would give a characteristic rupture length of $346.5 \mathrm{~km}$ (see Table 2).

The results of the calculation using the heterogeneous friction model on a fault of $2048 \times 16$ cells are shown in Figs. 4 6. As expected, for this friction model the slip pattern is much more complex than that of the smooth model. Distinct populations of large and small events are not apparent. In the development of slip during a single rupture nucleation is not as well defined as for the smooth model. Slip rapidly expands from the nucleation area. However, the average horizontal expansion velocity $\left(\bar{v}_{e} / c \sim 0.35\right)$ is in fact significantly slower than that of the smooth model (see Fig. 5). The reason for the low rupture velocity seems to be the high strength barriers of the heterogeneous friction model. As the rupture proceeds it builds up slip in an active region of a rupture and then jumps forward when sufficient slip has accumulated such that the boundary of the actively slipping subarea becomes unstable. As can be seen in Fig. 5, this process can lead to patchy propagation, where slip skips over high strength regions (or asperities) to form new patches, which may or may not propagate further.

Quake moment- and length-frequency distributions that result from heterogeneous model runs are shown in Figs. 6a and $b$. In both figures we obtain a powerlaw distribution of event sizes up to a roll-off point, where $N$ drops off exponentially. Here we are interested in finding the characteristic size associated with the change in statistics for large quakes. The heterogeneous model quake moment and length distributions are fit by the following modified GR distribution, which has previously been used to fit observed earthquake size distribu- 
(a)

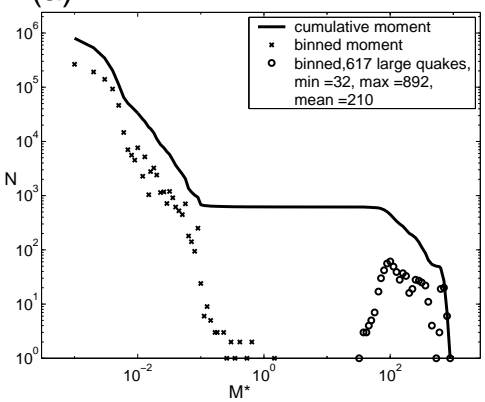

(c)

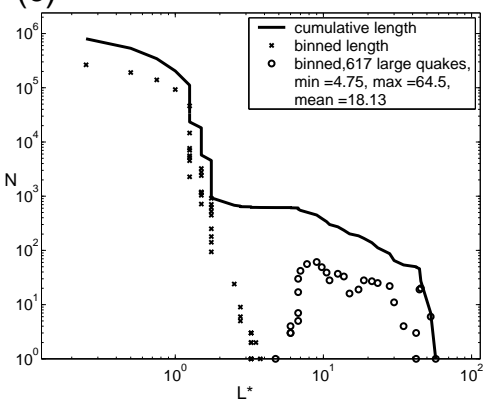

(b)

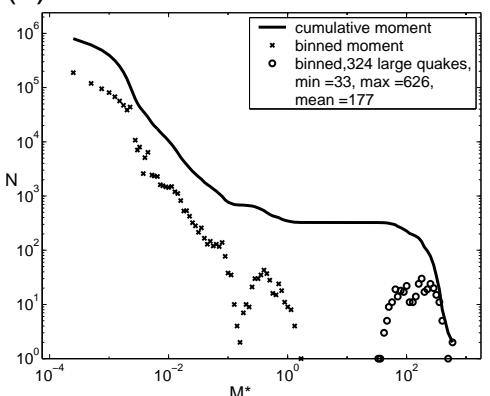

(d)

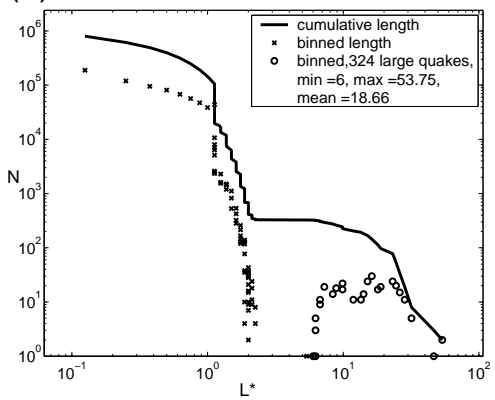

Fig. 8. Quake size distributions for the smooth fault model (compare with figure 7). Dimensionless Moment $\left(M^{*}\right)$ and dimensionless length $\left(L^{*}\right)$ distributions for two different aspect ratios. Figures $8 \mathrm{a}$ and $8 \mathrm{~b}$ are the moment size distributions for models with $\chi=256$ and $\chi=128$ respectively. Figures $8 \mathrm{c}$ and $\mathrm{d}$ are the length distributions for models with $\chi=256$ and $\chi=128$ respectively. tions (Utsu, 1999; and references therein; Kagan, 1997):

$N=\left(\frac{s}{\alpha}\right)^{-\beta} \exp \left[-\left(\frac{s}{\lambda}\right)^{\gamma}\right]$

where $N$ is the number of events $\geq$ size $s$ (which may be either moment or length), $\beta$ is the slope of the GR part of the distribution, $\lambda$ is the roll-off point of $s$ and $\gamma$ gives the steepness of the exponential roll-off.

\subsection{Faults of various aspect ratios}

To save calculation time, several model runs were performed with a 1-D row of slip cells. Instead of discretizing a vertical fault plane into a 2-D grid of square slip cells, the model fault is represented by a single horizontal row of slip cells, which are elongated in the vertical direction. As with 2-D fault grids, shear stresses for the 1-D faults are calculated at cell centers. While slip is laterally variable over the 1-D model fault, it is constant over individual cells. Thus, whereas slip is variable over depth in the 2-D models, it is constant in depth in the 1-D models. Nevertheless, it is found that the quake size distribution results for the 1-D faults are consistent with the results for the 2-D faults (see Table 2). This is expected for large events with horizontal extent significantly greater than vertical width since, in that case, a rupture on a 2-D fault expands mostly laterally, similar to a rupture on a 1-D fault.

Several calculations were performed for various fault aspect ratios. For both the heterogeneous and smooth friction models, we find that the characteristic rupture size does not vary significantly as a function of the length of the fault (see Figs. 7-9 and Table 2), as long as the characteristic rupture length is sufficiently small compared to the total fault length. Figure 7 shows the spatio-temporal slip patterns for models with $\chi=256$ (Fig. 7a) and $\chi=128$ (Fig. 7b). It is apparent that, for these models, changing the aspect ratio has a negligible effect on the characteristic dimensionless rupture size. This means that the quake length scales with the vertical fault width $h$ for these models. It is noted that, as the aspect ratio is decreased, rupture size scaling is strongly affected when the total fault length approaches about $3 \mu_{L^{*}}$, where $\mu_{L^{*}}$ is the mean dimensionless rupture length (recall that $L^{*}=L / h$ ). For fault lengths shorter than $3 \mu_{L^{*}}$, the system commonly locks into a sequence of rupture events that traverse the entire fault length; this behaviour for short faults is a result of the periodic boundary condition.

A similar characteristic rupture size distribution scaling is also apparent for the heterogeneous friction. This may be seen in Table 2. The characteristic dimensionless rupture length $\lambda_{L^{*}}$ has a range that is shown to be relatively constant over all of the calculations, representing aspect ratios $\chi=32,64$ and 128. Figure 9 shows the spatio-temporal slip distribution and the quake size distribution for the heterogeneous model with $\chi=128$. The relative constancy of $\lambda_{L^{*}}$ over a wide range of fault aspect rations implies that the change in scaling from powerlaw (GR) statistics to an exponential tail for large events is controlled by the width rather than the length of these model faults.

\section{Discussion}

As mentioned in the introduction, earthquake size distributions are characterized by a relatively constant powerlaw 

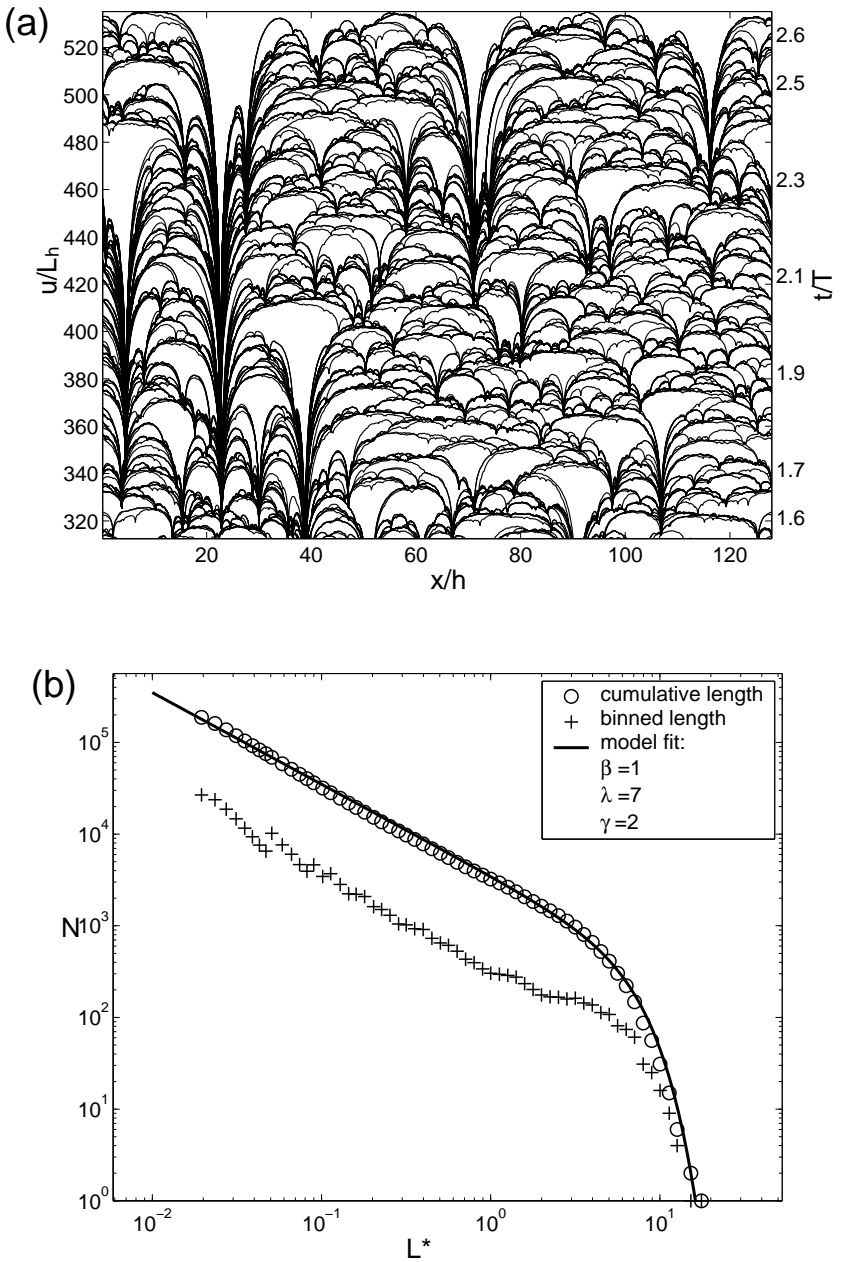

Fig. 9. Slip pattern and quake size distributions for the heterogeneous fault model. The fault plane is composed of $1024 \times 1$ rectangular cells. Figure 9a shows the spatio-temporal slip pattern. Figure $9 \mathrm{~b}$ shows the cumulative length distribution. For data interval and bin information see Fig. 3. The length (as well as moment) distributions for different aspect ratios and different grid geometries are roughly similar for the larger events. This shows that these models are independent of the size and shape of the model grid for the larger quakes; the fault depth sets the scale of the larger events.

slope that steepens for larger events. Typically $\beta \simeq 2 / 3$ corresponds to $b \simeq 1$ for a wide range of moderate earthquake sizes, where $\beta$ is the moment-frequency scaling parameter and $b$ is the GR or magnitude-frequency scaling parameter. The value of $\beta$ for large earthquakes is predicted by geometrical earthquake scaling models (Scholz, 1994; Heimpel, 1996). Given the condition of scale invariance, the values of $\beta$ are implied by the scaling between earthquake moment and area, $M \propto A^{1 / \beta}$. For a rupture expanding in two dimensions self-similarity implies that $\beta=2 / 3$, whereas for a rupture propagating horizontally, different models give different values of $\beta$. For the "W-model" of Romanowicz (1993), $\beta=1.0$ whereas for the "L-model" of Scholz (1994), $\beta=0.5$. The results presented here are consistent with the latter model.

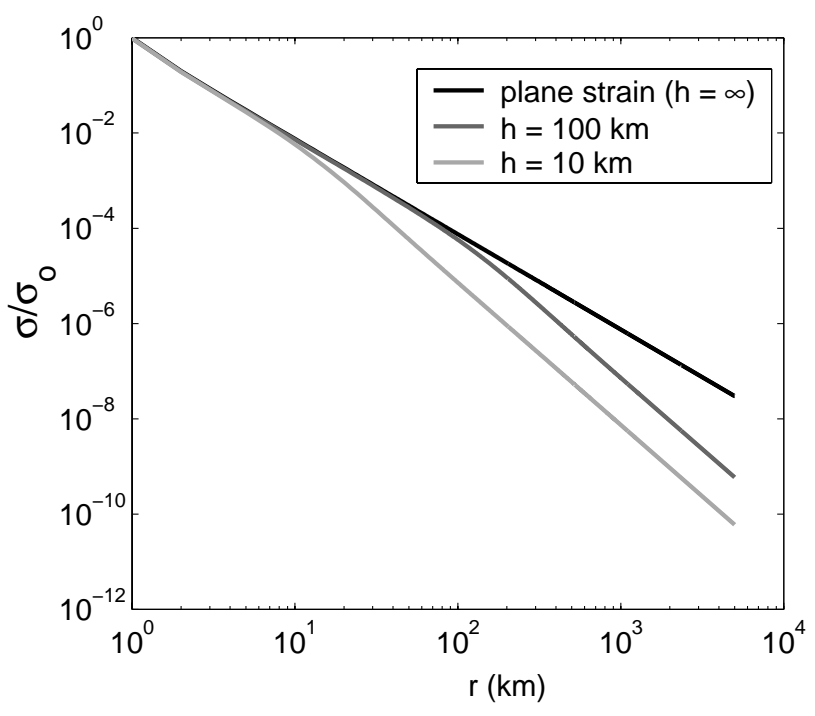

Fig. 10. Normalized stress ahead of a slip discontinuity with constant displacement across faults of three different vertical widths (denoted by $h$ ). The shear stress $\sigma$ is normalized by $\sigma_{o}$, the shear stress at a distance $r=1 \mathrm{~km}$ outside the rupture boundary. The faults of finite width extend from the free surface to depth $h$. Stress $\sigma$ is at a depth of $h / 2$.

Table 2 shows that for the heterogeneous friction model, $\beta=0.7$ for the $2-\mathrm{D}$ fault $(1024 \times 16$ cells $)$, whereas $\beta=0.5$ is typical for the $1-\mathrm{D}$ faults $(1024 \times 1$ cells $)$.

The foregoing discussion addresses only changes in earthquake size-frequency scaling due to geometrical considerations. The more profound scaling effect is a result of the detailed stress field due to fault slip (see Fig. 15 and discussion below). The models presented here are consistent with the "L-model" (we obtain powerlaw scaling with $\beta=0.5$ for small events). However, considering the full range of event sizes, the transition from small to large ruptures is accompanied by exponential roll off which increases the apparent value of $\beta$. Thus, although the L-model may be valid, an intermediate transition from $\beta \simeq 2 / 3$ to $\beta \simeq 1 / 2$ may be masked by the more severe exponential steepening of the distribution. We have interpreted $\lambda_{L^{*}}$ as marking the transition from small to large in earthquake length, where the subscript $L^{*}=L / h$ refers to the dimensionless length. The result for the 2-D model, of $\lambda_{L^{*}}=7.0$ (Table 2) is roughly consistent with the result of Yin and Rogers (1996). They obtained a scaling transition, which they called the "crossover length" for a rupture length of approximately 5 times the rupture width (corresponding to $\lambda_{L^{*}}=5$ here). The results here also bear a resemblance to the results of the rule-based numerical models of Gross (1996). There, the author found that heterogeneous models produced area versus slip scaling intermediate between the "L-model" and the "W-model".

The scaling of rupture events in this study may be understood in terms of the relationship between fault width and quasi-static stress transfer. Figure 15 shows the shear stress as a function of distance from the tip of a long slip discon- 
tinuity. Three cases are shown representing different fault widths; (1) plane strain (i.e. $h=\infty$ ), (2) $h=100 \mathrm{~km}$, and (3) $h=10 \mathrm{~km}$. For each of the cases the fault consists of a 1-D array of 2000 rectangular cells of width $h$ and length $l=1 \mathrm{~km}$, such that the total fault length is $2000 \mathrm{~km}$. The corresponding aspect ratios are: for case (1) $\chi=0$; case (2) $\chi=20$; and case (3) $\chi=200$. Shear stress $\sigma$ is taken at mid-depth and is normalized by $\sigma_{0}$, the stress at a horizontal distance $r=1 \mathrm{~km}$ (i.e. one cell length) from the rupture tip. In the far field for 2-D elasticity (i.e. plane strain) or at intermediate distances for 3-D elasticity, $\sigma$ falls off as $1 / r^{2}$. In the far field for 3-D elasticity $\sigma \sim 1 / r^{3}$. The distance of the break in scaling is proportional to the fault depth $h$. We can quantify the difference between the 2-D and 3-D cases by integrating the stress from the tip of the slip discontinuity to infinity for the case of plane strain and for a case with finite $h$. The difference between the two results is then a measure of the stress that is removed through the bottom boundary into the sub-surface. Hence the finite fault depth introduces stress dissipation that is proportional to $1 / h$. In fact, a simple way to introduce dissipation into a non-dissipative elastic system (e.g. plane strain or plane stress) is to define a scalar dissipation parameter that takes stress out of the system in proportion to the cumulative slip. Indeed, scalar dissipation parameters have been used in various kinds of earthquake models, including elastic models (Heimpel 1997) and rule based cellular automata (Steacy and McClosky, 1999).

\section{Summary}

We have studied several numerical models of repeating earthquakes on vertical planar faults. The faults are discretized either as a rectangular array of square slip cells (2-D fault) or a single row of rectangular slip cells (1-D fault). Two different fault rheologies are used: (1) a smooth model that results in populations of large events, with a well-defined mean size, and quasi-periodic recurrence; (2) a heterogeneous model resulting in powerlaw distribution of events that are well fit by modified GR distributions with $b \simeq 1$. For both models it is found that the characteristic scale of quakes is set by the vertical width of the seismogenic layer.

The smooth models produce two distinct populations of quakes; a set of large events and a set of much smaller events that add a negligible amount of moment release to the total. The large events have well defined mean moment and rupture length, which are both proportional to the fault depth. The characteristic size is defined by the mean sizes $\mu_{L^{*}}$ and $\mu$ for the dimensionless rupture length and dimensionless moment respectively.

The size distributions produced by the heterogeneous models are adequately fit by a three-parameter Gamma distribution. The characteristic size associated with the heterogeneous model is defined by scaling parameters $\lambda_{L^{*}}$ and $\lambda$ for the dimensionless rupture length and dimensionless moment respectively. The negative slope of the rupture length powerlaws for both $1-\mathrm{D}$ and $2-\mathrm{D}$ faults is $\beta_{L^{*}} \simeq 1$. The moment distributions for heterogeneous 1 -D faults yield $\beta \simeq 1 / 2$ for quakes of a small to moderate size. The size frequency distributions for quakes of dimensionless rupture length $L^{*} \geq 7.5$ fall off the GR distribution exponentially, such that, for the heterogeneous models $\lambda_{L^{*}} \simeq 7.5$. The moment distribution for the 2-D heterogeneous fault gives $\beta=0.7$ for quakes of small to moderate size.

The fault constitutive models discussed in the paper are meant to represent end-member models that give results spanning a range of fault behaviour. The smooth models result in characteristic earthquakes with a narrow range of large earthquake sizes. The heterogeneous models yield powerlaw size-frequency scaling up to a characteristic size, and exponential roll-off for larger events. As discussed in the introduction, earthquake catalogs exhibit a range of frequencysize distributions, depending on the fault system or even the fault segment represented in the catalogue and the time period of observation. This variability in observations has lead to different theories and models of earthquake behaviour. It is increasingly clear that real faults exhibit both self-similarity as well as the existence of characteristic length scales, again depending on the subset of data observed. Thus, the individual characteristics of earthquake size-frequency distribution can potentially be used to guide the identification observable length scales associated with faults and fault systems.

Acknowledgements. The author thanks Leon Knopoff for support at UCLA. The comments of John McCloskey and an anonymous referee are gratefully acknowledged. Partial financial support was provided by NSERC.

\section{References}

Andrews, D. J.: A stochastic fault model, 1, Static case, J. Geophys. Res., 85, 3867-3877, 1980.

Andrews, D. J.: A fault constitutive relation accounting for thermal pressurization of pore fluid, J. Geophys. Res., 107, 2363, 2002.

Beeler, N. M., Tullis, T. E., and Weeks, J. D.: The roles of time and displacement in the evolution effect in rock friction, Geophys. Res. Lett. 21, 1987-1990, 1994.

Ben-Zion, Y. and Rice, J. R.: Earthquake failure sequences along a cellular fault zone in a three-dimensional elastic solid containing asperity and nonasperity regions, J. Geophys. Res., 98, 14 109$14131,1993$.

Burridge, R. and Knopoff, L.: Model and theoretical seismicity, Bull. Seismol. Soc. Am., 57, 347, 1967.

Carlson, J. M. and Langer, J. S.: Mechanical model of an earthquake fault, Phys. Rev. 40, 6470-6484, 1989.

Dieterich, J. H.: Modeling of rock friction 1. experimental results and constitutive equations. J. Geophys. Res. 84, 2161-2168, 1979.

Dieterich, J. H.: Earthquake nucleation on faults with rate- and state-dependent strength, Tectonophysics, 211, 1992.

Gross, S.: Magnitude distributions and slip scaling of heterogeneous seismic source, Bull. Seismol. Soc. Am., 86, 498-504, 1996.

Groves, P. P. and Bacon, D. J.: The dislocation loop near a free surface, Phil. Mag., 22, 83-91, 1970.

Heaton, T. H.: Evidence for and implications of self-healing pulses of slip in earthquake rupture, Phys. Earth Planet. Interiors, 64, 1-20, 1990. 
Heimpel, M.: Earthquake size-frequency relations from an analytical stochastic rupture model, J. Geophys. Res., 101, 22 43522 448, 1996.

Heimpel, M.: Critical behaviour and the evolution of fault strength during earthquake cycles, Nature 388, 865-868, 1997.

Hirth, J. P. and Lothe, J.: Theory of Dislocations, John Wiley and Sons, Publishers, 1982.

Hyndman, R. D. and Weichert, D. H.: Seismicity and rates of relative plate boundaries of Western North America, Geophys. J. R. Astr. Soc. 72, 59-82, 1983.

Kagan, Y. Y.: Seismic moment-frequency relation for shallow earthquakes: Regional comparison, J. Geophys. Res. 102, 2835-2852, 1997.

Lapusta, N., Rice, J. R., Ben-Zion, Y., and Zheng, G. T.: Elastodynamic analysis for slow tectonic loading with spontaneous rupture episodes on faults with rate- and state-dependent friction, J. Geophys. Res., 105, 23 765-23 789, 2000.

Maurrisson, Y. and Capella, L.: Stress field of a dislocation segment perpendicular to a free surface, Phil. Mag., 29, 679-683, 1974a.

Maurrisson, Y. and Capella, L.: Stress field of a dislocation segment parallel to a free surface, Phil. Mag., 29, 1227-1229, 1974b.

Nielsen, S. B. and Knopoff, L.: The equivalent strength of geometrical barriers to earthquakes. J. Geophys. Res., 103, 9953-9965, 1998.

Okal, E. A. and Romanowicz, B. A.: On the variation of $b$-values with earthquake size, Phys. Earth Planet. Inter., 87, 55-76, 1994.

Pacheco, J., Scholz, C., and Sykes, L.: Changes in frequencymagnitude relationship from small to large earthquakes, Nature, 355, 71-73, 1992.

Rice, J. R.: Spatio-temporal complexity of slip on a fault. J. Geophys. Res. 98, 9885-9907, 1993.

Rice, J. R. and Ben-Zion, Y.: Slip complexity in earthquake fault models, Proc. Natl. Acad. Sci. 93, 3811-3818, 1996.
Romanowicz, B.: On scaling relations for large earthquakes, Bull. Seismol. Soc. Am., 83, 1294-1297, 1993.

Rundle, J.: Derivation of the complete Gutenberg-Richter magnitude-frequency relation using the principle of scale invariance, J. Geophys. Res., 94, 12 337-12 342, 1989.

Scholz, C. H.: A reappraisal of large earthquake scaling, Bull. Seismol. Soc. Am., 84, 215-218, 1994.

Scholz, C. H., Wyss, M., and Smith, S. W.: Seismic and aseismic slip on the San Andreas Fault, J. Geophys. Res. 74, 2049-2069, 1969.

Schwartz, D. P. and Coppersmith, K. J.: Fault behavior and characteristic earthquakes: examples from the Wasatch and San Andreas Fault zones. J. Geophys. Res. 89, 5681-5698, 1984.

Shaw, B. E.: The edges of large earthquakes and the epicenters of future earthquakes: Stress induced correlations in elastodynamic fault models, Pure appl. geophys. 157, 2149-2164, 2000.

Sieh, K.: The repetition of large earthquake ruptures, Proc. Natl. Acad. Sci. 93, 3764-3771, 1996.

Sleep, N. H.: Application of a unified rate and state friction theory to the mechanics of fault zones with strain localization, J. Geophys. Res., 102, 2875-2895, 1997.

Steacy, S. and McCloskey, J.: Heterogeneity and the earthquake frequency-magnitude distribution, Geophys. Res. Lett. 26, 899902, 1999.

Utsu, T.: Representation and analysis of the earthquake size distribution: A historical review and some new approaches, Pure Appl. Geophys. 155, 509-535, 1999.

Wesnousky, S. G.: The Gutenberg-Richter or characteristic earthquake distribution, which is it? Bull. Seis. Soc. Am., 84, 19401959, 1994.

Yin, Z. M. and Rogers, G. C.: Toward a physical understanding of earthquake scaling relations, Pure Appl. Geophys., 146, 661675, 1996. 